\title{
Stability of Existing Banyukuwung Dam in Recent Hydrology and Geotechnical Conditions
}

\author{
Pitojo Tri Juwono $^{1}$, Runi Asmaranto ${ }^{1^{*}}$, Ari Murdhianti ${ }^{2}$ \\ ${ }^{1}$ Water Resources Engineering Department, Faculty of Engineering, Universitas \\ Brawijaya, Malang, 65145, Indonesia \\ ${ }^{2}$ Associate Expert water resources and dam, PT. Triando Konsultama, Malang, 65145, \\ Indonesia \\ runi_asmaranto@ub.ac.id
}

Received 08-04-2019; accepted 25-06-2020

\begin{abstract}
Banyukuwung Dam is located in the villages of Sukorejo and Sudo, Sumber District, Rembang Regency, Central Java Province. This dam was built in 1995-1997 to serve the needs of 7750 ha of irrigation water and $35 \mathrm{l} / \mathrm{s}$ of raw water needs. Dam type is homogeneous reservoir, has a height of $19.40 \mathrm{~m}$ above the riverbed and $25.40 \mathrm{~m}$ above the foundation excavation. The length of the dam peak is $181.00 \mathrm{~m}$ and the width is $5.00 \mathrm{~m}$ while the reservoir volume under normal water conditions is 1.64 million $\mathrm{m} 3$. Along with seasonal changes and extreme hydrological behavior and based on an investigation of current geotechnical conditions, stability analysis is needed based on these two conditions. It is very important to plan operational and maintenance activities related to the dam maintenance program. So that it is expected to be useful for the relevant agencies in making operational decisions. The purpose of this study was to determine the stability of the existing Banyukuwung dam based on the latest hydrological and geotechnical behavior. The results are expected to provide recommendations in the management, operation and maintenance of the dam manager
\end{abstract}

Keywords: Banyukuwung reservoir, dam stability, hydrogeotechnical

\section{Introduction}

Water Resources are very important for humans because they are basic human needs. From all of the water on the surface of the earth, only $2.5 \%$ is in the form of fresh water. With this very limited amount, then needs the presence of protection on the existence of water resources and their optimal use. In the context of the maximum utilization of water sources, needed the development effort and good water management namely by building reservoirs to store water.

Banyukuwung Dam is located in Sumber District, Rembang Regency, it was built in 1995 [2]. In general, the Dam is to collect water that is used to meet various needs including irrigation, raw water, flood control, tourism and others. Analysis of the existing Banyukuwung dam stabilization needs to be done considering the age of the building which is old and there are some symptoms of damage due to hydraulic behavior, so that the preservation of building functions will be maintained [1][2][3].

Cite this as: Juwono, P., Asmaranto, R., \& Murdhianti, A. (2020). Stability of Existing Banyukuwung DAM in Recent Hydrology and Geotechnical Conditions. Civil and Environmental Science Journal (Civense), 3(2), 6071. doi: https://doi.org/10.21776/ub.civense.2020.00302.1 


\section{Material and Methods}

\subsection{Study Location}

The Study Location is Banyukuwung Dam which is located at the coordinate $06^{\circ} 46^{\prime} 51^{\prime \prime} \mathrm{S}$ and $111^{\circ} 19^{\prime} 18,9^{\prime \prime}$ E in Banyukuwung, Sukorejo and Sudo Village, Sumber Subdistrict, Rembang Regency, Central Java Province.
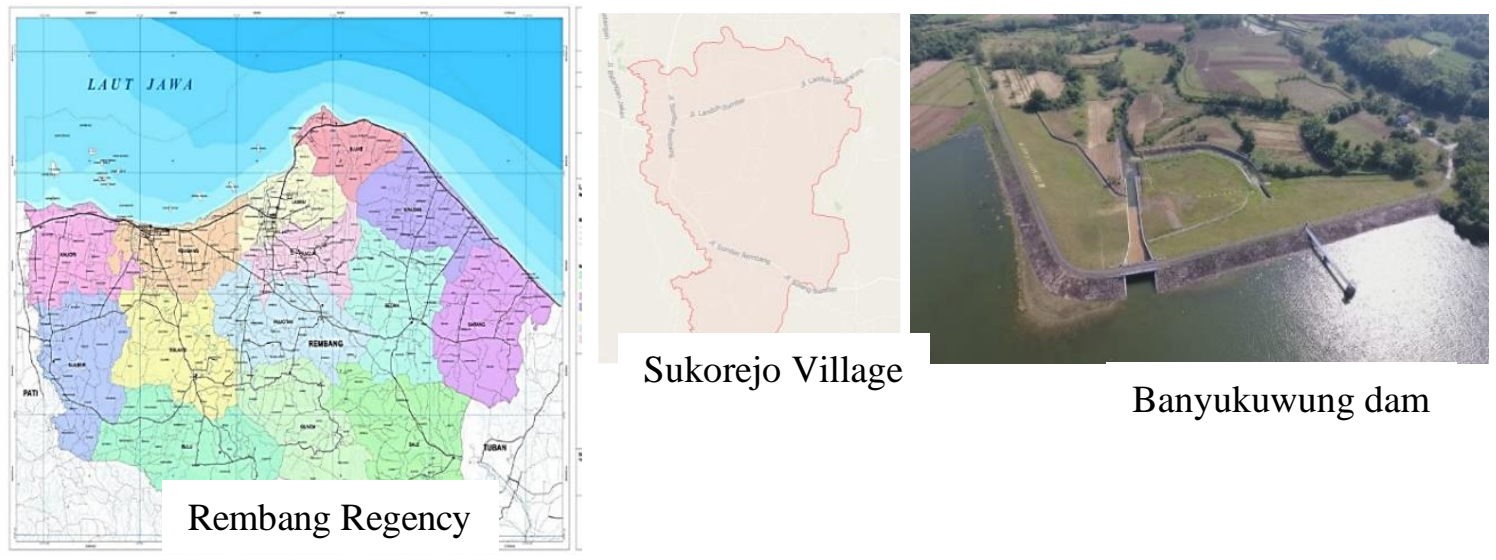

Figure 1. Location of Study

Banyukuwung Dam was built to provide 35 1/s raw water estimated to be able to meet the water needs of $\pm 20,000$ residents in Rembang Regency, especially Kaliori District; and to provide irrigation supplies of around 775 ha through the Pentil Weir [2].

\subsection{Primary and secondary data collection}

Data needed in the processing of this study, among others [2][5];

1. Rainfall Data from Banyukuwung station for 1973-2017

2. Map of the Indonesian Earthquake 2017

3. Geotechnical investigation data of the foundation and body of Dam

4. Cross section of Banyukuwung dam body

5. Instrumentation measurement of standpipe Piezometer

\subsection{Study Stage}

The steps of this study are arranged systematically so that facilitate the completion of this analysis. The steps of the study that conducted are as follows [5][6];

1. Primary and secondary data collection

2. Determine the regional rainfall

3. Test the skewness of rainfall data

4. Frequency analysis test

5. Goodness of fit test

6. Maximum annual daily rainfall

7. Analysis of design flood discharge with the latest hydrological conditions

8. Flood Routing

9. Analysis of seepage in the body of the dam using the latest geotechnical data

10. Analysis of dam body stability using Bishop modified methods uses the latest soil sampling data

\subsection{Geotechnical Survey and Investigation}

A geotechnical investigation survey was conducted to find out the latest geotechnical parameters which include [5][11] 
1. Piezometer measurement

2. Investigation of undisturbed sampling at :

a. physical properties of landfill and foundation

b. mechanical properties of soil embankments and foundations

c. Permeability of embankments and foundation. Investigation of land in the field was carried out by PT Supraharmonia in collaboration with the dam manager. The location of undisturbed sampling points is as follows:

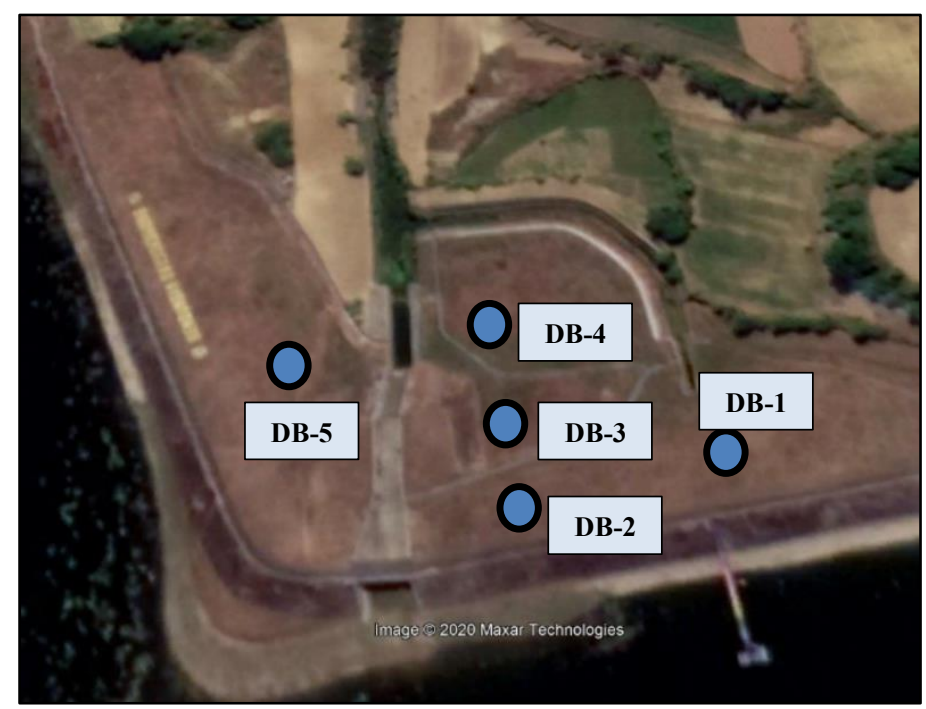

Figure 2. Drilling Bor location of soil properties investigation

Table. 1. Coordinate of drilling bore and depth

\begin{tabular}{|c|c|c|c|}
\hline Location & $\mathbf{X}$ & $\mathbf{Y}$ & Depth (m) \\
\hline DB-1 & 535460.61 & 9250423.01 & $15 \mathrm{~m}$ \\
\hline DB -2 & 535403.03 & 9250413.43 & $15 \mathrm{~m}$ \\
\hline DB -3 & 535398.74 & 9250439.85 & $10 \mathrm{~m}$ \\
\hline DB -4 & 535396.65 & 9250459.20 & $10 \mathrm{~m}$ \\
\hline DB -5 & 535327.03 & 9250448.97 & $10 \mathrm{~m}$ \\
\hline
\end{tabular}

5 (five) drilling point locations (2@15 m and $3 @ 10 \mathrm{~m}$ accompanied by a standard penetration test) have been carried out accompanied by fairly undisturbed samples in several depths of soil.

\subsection{Hydrological Analysis}

Hydrological analysis is done by comparing the analysis of rainfall and flood plans based on the results of analysis of studies in 1999, 2015 and present conditions. Hydrological calculations use basic rain data at Sulang and Sumber rain stations. Flood tracing is conducted to determine the modification of flood flow, to find out the maximum outflow discharge and maximum water level above the overflow threshold.

Frequency analysis can be applied to river discharge or rain data using annual maximum data, the largest data that occurs during one year, measured over several years. There are several forms of continuous (theoretical) distribution functions, which are often used in frequency analysis for hydrology such as the Normal distribution, Normal Log, Gumbel, Pearson, Pearson Log, etc [8][15]. 


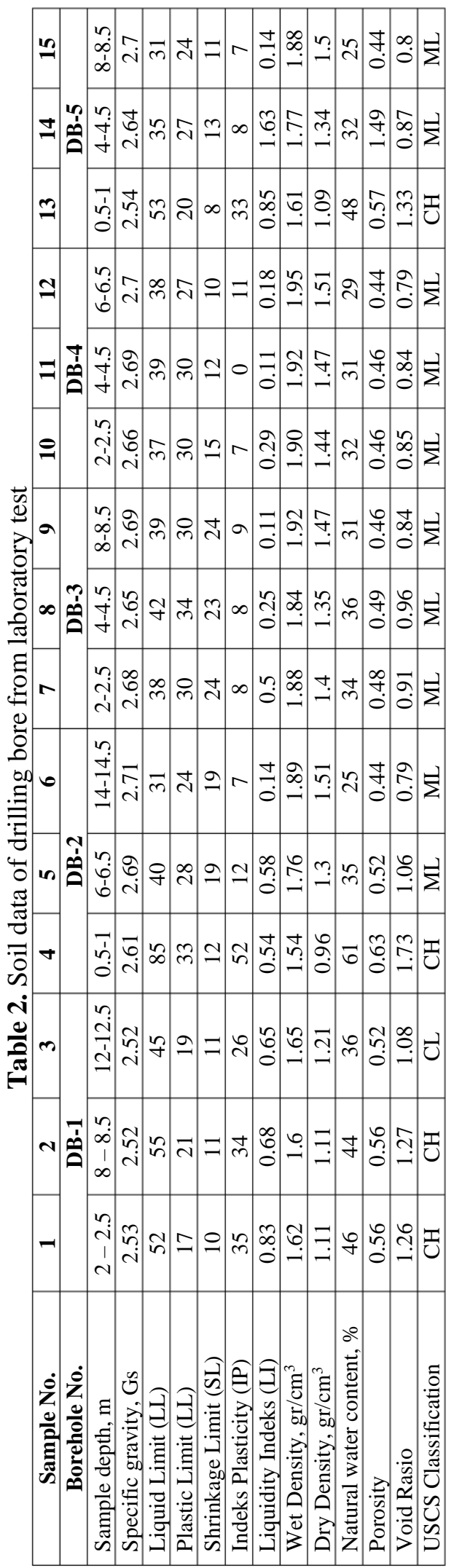

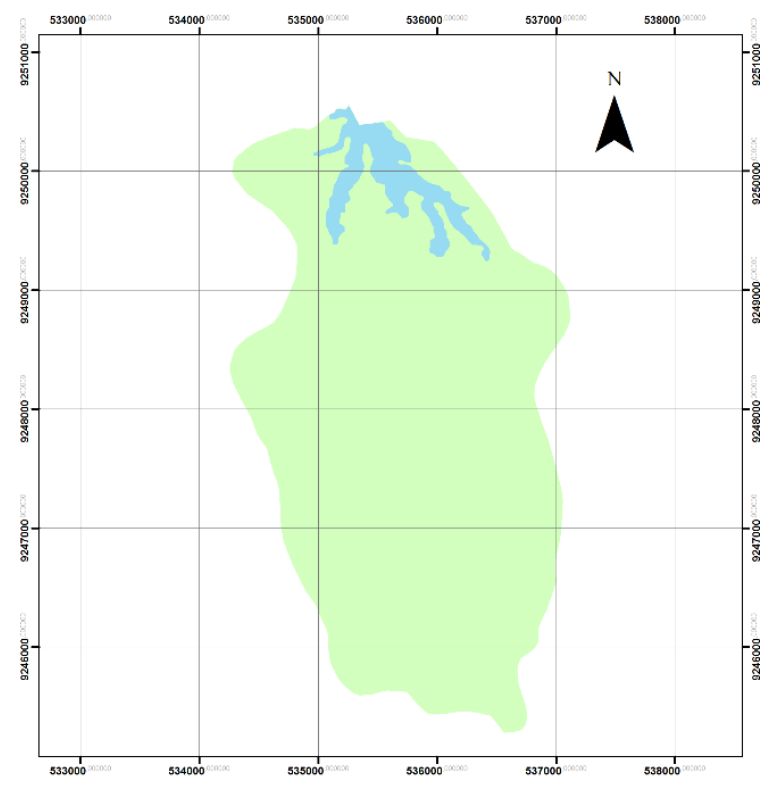

Figure 3. Banyukuwung catchment area $\left(11.20 \mathrm{~km}^{2}\right)$

\subsection{Design Flood Discharge}

Design Flood Discharge is a flood discharge used to plan the safety level of danger with the largest probability number. To analyze the design flood discharge can be done using the hydrograph method which is conducted using the assist of a synthetic unit hydrograph model and non-hydrographic methods carried out with the help of frequency analysis techniques.

In this study conducted the comparison of Nakayasu Synthetic Unit Hydrograph method [17], Gamma I Synthetic Unit Hydrograph [16] and HSS ITB Synthetic Unit Hydrograph method [6][8]

\subsection{Flood Routing}

To obtain the flood water level on the dam body it is necessary to conduct flood routing to determine the outflow discharge of the flood storage in the reservoir [11][17].

The width threshold type spillway is used with elevation and volume as follows [4][12];

$\mathrm{Q}=\mathrm{Cd} \times \mathrm{B} \times \mathrm{H}^{3 / 2}$

With:

$\mathrm{Cd}=$ Discharge coefficient

$\mathrm{B}=$ Diversion width $(\mathrm{m})$

$\mathrm{H}$ = Water Level above the Spillway (m) 
In many cases, to build a fill type dam it is expected to be able to calculate the stability of the talud in order to check the safety of the natural talud, the cut talud, and the fill talud obtained. [1][2]. To determine the condition of the stability of the dam slope, used the modified Bishop Method. Bishop introduced a more thorough solution than a simple slice method. In this method, the effect of the forces on the edge of each slice is taken into account, as follows [3][4][5][14].

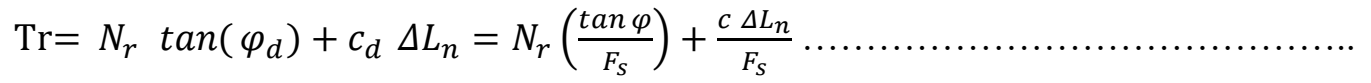

$$
\begin{aligned}
& N r=\frac{W_{n}+\Delta T-\frac{c \Delta L_{n}}{F_{S}} \sin \alpha_{n}}{\cos \alpha_{n}+\frac{\tan \varphi \sin \alpha_{n}}{F_{S}}} \\
& F S=\frac{\sum_{n=1}^{n=p}\left(c b_{n}+W_{n} \tan \varphi\right) \frac{1}{m_{\alpha(n)}}}{\sum_{n=1}^{n=p} W_{n} \sin \alpha_{n}}
\end{aligned}
$$

\subsection{Stability against Seepage}

Seepage through dam bodies, foundations, abutment, or hills around the reservoir must be controlled, so that excessive uplift force may not occur.

The safety of soil fill type dam can be calculated based on the following formula [5]; $F s=\frac{I_{c}}{I_{e}} \geq 4$

\section{Result and Discussion}

\section{1. Rain storm and Design Flood}

Based on the results of frequency analysis based on rainfall data at Banyukuwung Station, obtained rainfall design and design flood as follows:

Table 3. Recapitulation of the calculation results of Rain storm - Design Flood - Return period ( $\mathrm{Tr}$ )

\begin{tabular}{|c|c|c|c|c|c|c|c|c|}
\hline \multirow{2}{*}{$\operatorname{Tr}$} & \multicolumn{4}{|c|}{ Rain storm } & \multicolumn{4}{c|}{ Design flood } \\
$\mathrm{R}(\mathrm{mm})$ & & \multicolumn{4}{c|}{} \\
\cline { 2 - 9 } & 1999 & 2015 & 2016 & 2019 & 1999 & 2015 & 2016 & 2019 \\
\hline 10 & 140.77 & 98.08 & 108.91 & 99.57 & 140.77 & 98.08 & 108.91 & 99.57 \\
\hline 100 & 216.07 & 114.64 & 127.75 & 124.68 & 216.07 & 114.64 & 127.75 & 124.68 \\
\hline 1000 & 308.18 & 126.75 & 141.53 & 140.71 & 308.18 & 126.75 & 141.53 & 140.71 \\
\hline PMP & 700 & 315.53 & 317 & 260.60 & 700 & 315.53 & 317 & 260.60 \\
\hline
\end{tabular}

Based on the above analysis, it can be seen that there was a decrease in design flood discharge when compared to the data ranges of the previous year (2015 and 2016). While the difference is significant in 1999, because the catchment area of the reservoir studied was different. Table 4 shows the results of the calculation of the rainfall and flood design with return periode each $\mathrm{T}=50,100$ and 1000 years. Moreover, it is also compared with condition of 0.5 PMF (probable maximum flood) and PMF. 


\begin{tabular}{|c|c|c|c|c|c|c|c|c|c|c|}
\hline Z & & & & & & & $\begin{array}{l}00 \\
\ddots \\
0 \\
0 \\
0 \\
0 \\
0 \\
0 \\
0 \\
0 \\
0\end{array}$ & and & & 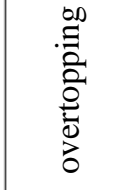 \\
\hline \multirow{8}{*}{ 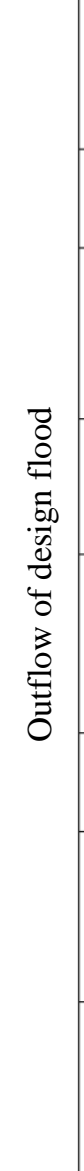 } & 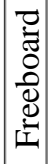 & $\Xi$ & $\exists$ & : & $\hat{\sigma}$ & $\underset{-}{\sigma}$ & 官 & $\begin{array}{l}8 \\
i \\
i\end{array}$ & $\stackrel{さ}{\stackrel{\Xi}{ }}$ & 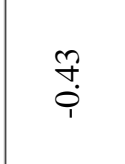 \\
\hline & 품 & $\Xi$ & ๗ે? & $\stackrel{f}{\sim}$ & n? & $\stackrel{\infty}{+}$ & $\begin{array}{c}\tilde{f} \\
\dot{v}\end{array} \mid$ & $\begin{array}{l}\stackrel{P}{q} \\
\dot{r}\end{array}$ & ণั & $\hat{\sim}$ \\
\hline & 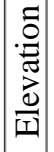 & $\Xi$ & $\begin{array}{l}2 \\
\infty \\
\text { in }\end{array}$ & 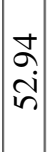 & $\begin{array}{l}\hat{0} \\
\dot{n} \\
\dot{n}\end{array}$ & 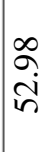 & $\begin{array}{l}\hat{\sigma} \\
\hat{n}\end{array}$ & $\begin{array}{l}0 \\
0 \\
n\end{array}$ & $\frac{0}{i}$ & $\begin{array}{l}\stackrel{9}{+} \\
\dot{f}\end{array}$ \\
\hline & 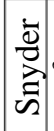 & 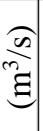 & $\frac{\mathcal{r}}{\stackrel{\gamma}{\gamma}}$ & $\begin{array}{l}\vec{J} \\
\stackrel{+}{q}\end{array}$ & $\begin{array}{l}n \\
n \\
\vdots \\
n\end{array}$ & $\frac{\infty}{n}$ & 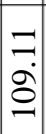 & 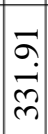 & $\frac{\text { qे. }}{\infty}$ & $\begin{array}{l}\stackrel{m}{\sim} \\
\infty \\
\infty \\
\infty\end{array}$ \\
\hline & 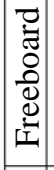 & $\Xi$ & $\stackrel{\infty}{\stackrel{\infty}{0}}$ & $\stackrel{N}{\stackrel{N}{0}}$ & ڤn & : & 守 & $\stackrel{\widehat{N}}{\stackrel{\gamma}{i}}$ & $\stackrel{n}{0}$ & $\stackrel{m}{\rightarrow}$ \\
\hline & 푼 & $\Xi$ & $\stackrel{N}{\sim}$ & $\stackrel{\infty}{\underset{7}{-}}$ & ठே & 忍 & $\begin{array}{l}\hat{a} \\
\text { ì }\end{array}$ & $\stackrel{2}{\circ}$ & $\stackrel{n}{\check{2}}$ & $\underset{\dot{\sigma}}{\hat{r}}$ \\
\hline & 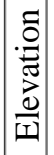 & $\Xi$ & $\begin{array}{l}\text { ते } \\
\text { ñ }\end{array}$ & $\begin{array}{l}\infty \\
\\
\\
\tilde{n}\end{array}$ & $\begin{array}{l}\text { z } \\
\dot{n} \\
\text { n. }\end{array}$ & $\begin{array}{l}\text { 寸 } \\
\text { ஜn }\end{array}$ & $\begin{array}{l}\dot{y} \\
\dot{v} \\
\dot{n}\end{array}$ & $\begin{array}{l}\hat{v} \\
\infty \\
n \\
n\end{array}$ & $\begin{array}{l}\stackrel{2}{n} \\
\text { nn }\end{array}$ & $\frac{m}{6}$ \\
\hline & 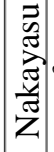 & $\frac{\partial}{3}$ & $\begin{array}{l}2 \\
\infty \\
\text { తి. }\end{array}$ & ?ָ & ڤ్ & ?ே & $\begin{array}{l}n \\
\dot{f} \\
\pm\end{array}$ & $\begin{array}{l}n \\
\stackrel{n}{g} \\
\stackrel{\vartheta}{q}\end{array}$ & $\begin{array}{l}\infty \\
\stackrel{\infty}{m} \\
\stackrel{m}{n}\end{array}$ & $\begin{array}{l}\text { m. } \\
\infty \\
\text { ले }\end{array}$ \\
\hline 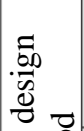 & 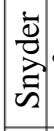 & $\stackrel{\Xi}{\Xi \Xi}$ & $\begin{array}{l}\text { ஜ } \\
\stackrel{0}{n} \\
\stackrel{n}{n}\end{array}$ & 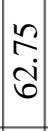 & $\begin{array}{c}2 \\
0 \\
0\end{array}$ & \begin{tabular}{l}
7 \\
$\dot{7}$ \\
\multirow{6}{*}{}
\end{tabular} & $\begin{array}{l}2 \\
\infty \\
\dot{0} \\
-1\end{array}$ & 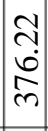 & $\begin{array}{l}\infty \\
\infty \\
\stackrel{0}{-}\end{array}$ & 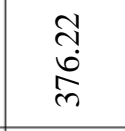 \\
\hline 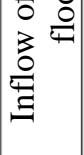 & 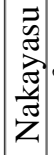 & 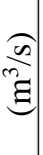 & $\begin{array}{l}\infty \\
\stackrel{0}{0} \\
=\end{array}$ & $\mid \begin{array}{l}n \\
0 \\
0 \\
0 \\
=\end{array}$ & $\begin{array}{l}n \\
\infty \\
\dot{m} \\
\end{array}$ & $\stackrel{\infty}{a}$ & 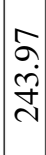 & 웅 & à & io \\
\hline 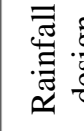 & & ఏ్త్ & $\begin{array}{l}\bar{n} \\
\infty \\
=\end{array}$ & $\begin{array}{l}\infty \\
0 \\
\dot{+} \\
\simeq\end{array}$ & 尺̃ & 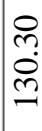 & 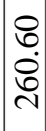 & $\begin{array}{l}8 \\
8 \\
\grave{0} \\
\end{array}$ & 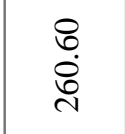 & $\begin{array}{l}\stackrel{0}{\circ} \\
\stackrel{2}{n}\end{array}$ \\
\hline- & & 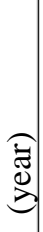 & & 8 & \& & $\begin{array}{l}\sum_{1}^{I} \\
\vdots \\
0 \\
0\end{array}$ & $\sum_{0}^{1}$ & 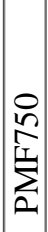 & 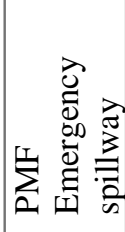 & 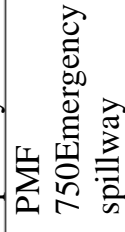 \\
\hline
\end{tabular}

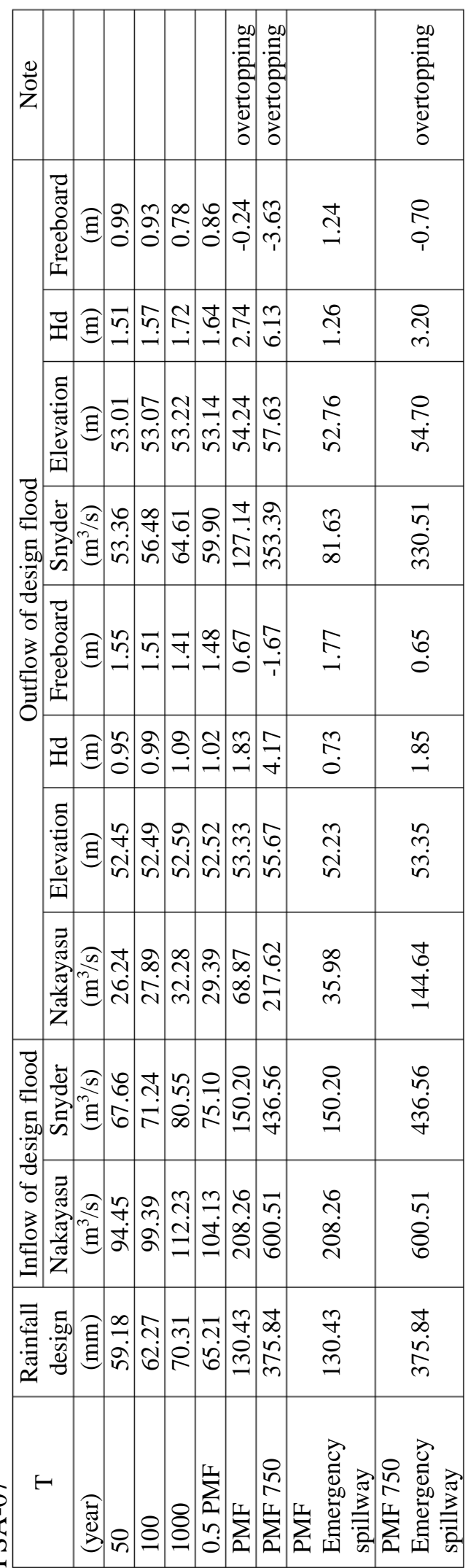




\begin{tabular}{|c|c|c|c|c|c|c|c|c|c|}
\hline$\left|\begin{array}{l}0 \\
0 \\
z\end{array}\right|$ & & & & & & & & $\begin{array}{l}\text {.0 } \\
.0 \\
0 \\
0 \\
0 \\
0 \\
0\end{array}$ & \\
\hline \multirow{8}{*}{ 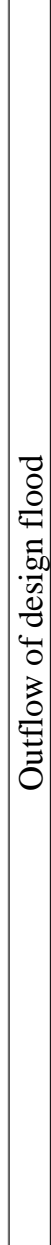 } & 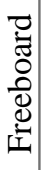 & $\Xi$ & $\begin{array}{l}\infty \\
0 \\
- \\
-\end{array}$ & تُ & $\stackrel{0}{n}$ & مِ & $\hat{a}$ & ণి & さ্さ \\
\hline & $\underset{I}{\mathbf{I}}$ & $\Xi$ & $\begin{array}{l}\widetilde{N} \\
0 \\
0\end{array}$ & ஜ & à & $\begin{array}{l}\infty \\
\infty \\
0\end{array}$ & $\stackrel{n}{n}$ & $\frac{0}{\stackrel{0}{r}}$ & ণิ ָุ \\
\hline & 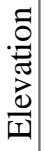 & $\Xi$ & 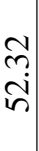 & $\begin{array}{l}\text { ָे } \\
\text { ñ } \\
\text { ñ }\end{array}$ & 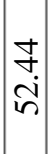 & $\begin{array}{l}\infty \\
\text { ñ. } \\
\text { ñ }\end{array}$ & $\begin{array}{l}0 \\
\dot{n} \\
\tilde{n}\end{array}$ & 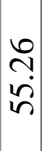 & $\frac{0}{i}$ \\
\hline & 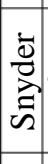 & 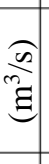 & 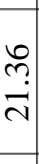 & $\begin{array}{l}\text { ర్ } \\
\text { त̇ }\end{array}$ & $\begin{array}{l}\infty \\
\infty \\
\\
2\end{array}$ & $\begin{array}{l}\stackrel{8}{0} \\
\ddot{\lambda}\end{array}$ & $\begin{array}{l}0 \\
0 \\
i \\
\text { n }\end{array}$ & $\begin{array}{l}\infty \\
\infty \\
\infty \\
\infty \\
\infty\end{array}$ & $\underset{\infty}{\stackrel{\infty}{\sim}}$ \\
\hline & 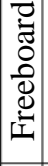 & $\Xi$ & ف‥ & $\stackrel{n}{2}$ & $\tilde{n}$ & $\underline{\sigma}$ & $\begin{array}{l}\infty \\
\vdots \\
0\end{array}$ & $\underset{7}{\sim}$ & $\stackrel{\text { m}}{-}$ \\
\hline & $\bar{T}$ & $\Xi$ & $\begin{array}{l}\bar{\infty} \\
\dot{0}\end{array}$ & $\stackrel{\mathscr{\infty}}{\infty}$ & $\hat{\varrho}$ & $\begin{array}{l}\infty \\
0\end{array}$ & กี & $\begin{array}{c}\tilde{\sigma} \\
\dot{r} \\
\text { r. }\end{array}$ & ণิ \\
\hline & 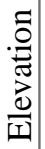 & હ્ડ & $\begin{array}{l}\bar{m} \\
\text { ñ. } \\
\text { ñ. }\end{array}$ & $\begin{array}{l}\text { ñ } \\
\text { ñ. } \\
\text { ñ }\end{array}$ & $\begin{array}{l}m \\
\stackrel{a}{+} \\
\stackrel{v}{n}\end{array}$ & ñ. & $\begin{array}{c}\tilde{O} \\
0 \\
\tilde{n} \\
\end{array}$ & $\begin{array}{l}\sim \\
\stackrel{2}{n} \\
\sim\end{array}$ & $\begin{array}{l}\text { 울 } \\
\text { in }\end{array}$ \\
\hline & 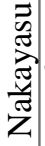 & $\stackrel{2}{m}$ & $\begin{array}{c}0 \\
\stackrel{\sim}{\mathrm{N}}\end{array}$ & $\begin{array}{l}\hat{2} \\
\text { } \\
\text { ते }\end{array}$ & $\begin{array}{l}m \\
\check{2} \\
\check{\sim}\end{array}$ & $\begin{array}{l}\infty \\
\text { ஸे } \\
\stackrel{\sim}{\sim}\end{array}$ & $\begin{array}{l}\hat{\sigma} \\
\text { in } \\
\text { n. }\end{array}$ & $\begin{array}{l}0 \\
\dot{0} \\
\dot{1} \\
\infty\end{array}$ & $\begin{array}{l}\stackrel{0}{\circ} \\
\stackrel{1}{r}\end{array}$ \\
\hline 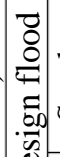 & 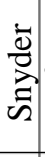 & $\frac{a}{2}$ & $\begin{array}{l}\stackrel{n}{n} \\
n \\
n\end{array}$ & $\begin{array}{l}\stackrel{\gamma}{t} \\
\stackrel{2}{\gamma}\end{array}$ & $\begin{array}{l}0 \\
0 \\
\infty \\
\infty\end{array}$ & $\begin{array}{l}\circ \\
\infty \\
\infty \\
\infty\end{array}$ & $\begin{array}{l}\mathcal{N} \\
\mathfrak{2} \\
0 \\
-0\end{array}$ & \begin{tabular}{c}
$\infty$ \\
+ \\
$\dot{v}$ \\
\multirow{\gamma}{\gamma}{} \\
\end{tabular} & 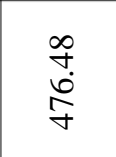 \\
\hline 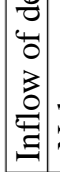 & 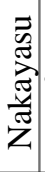 & $\stackrel{\text { (n) }}{\mathrm{m}}$ & 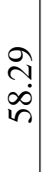 & ๘) & $\frac{0}{3}$ & $\begin{array}{l}\text { बे. } \\
\text { తై }\end{array}$ & 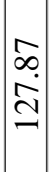 & $\begin{array}{l}n \\
n \\
6 \\
n \\
n\end{array}$ & $\begin{array}{l}n \\
n \\
\hat{n} \\
n\end{array}$ \\
\hline 胥 & 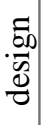 & ఏ્ఏ & $\begin{array}{l}\bar{n} \\
\infty \\
=\end{array}$ & $\begin{array}{l}\infty \\
0 \\
\dot{J} \\
\end{array}$ & 尺્ઞ & 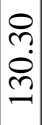 & 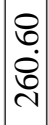 & $\begin{array}{l}0 \\
\dot{i} \\
\stackrel{n}{r}\end{array}$ & $\begin{array}{l}0 \\
\stackrel{0}{n} \\
r\end{array}$ \\
\hline- & & 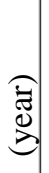 & & & 8 & $\begin{array}{l}n \\
n \\
0\end{array}$ & $\sum_{\Omega}^{\infty}$ & 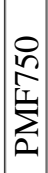 & 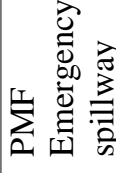 \\
\hline
\end{tabular}

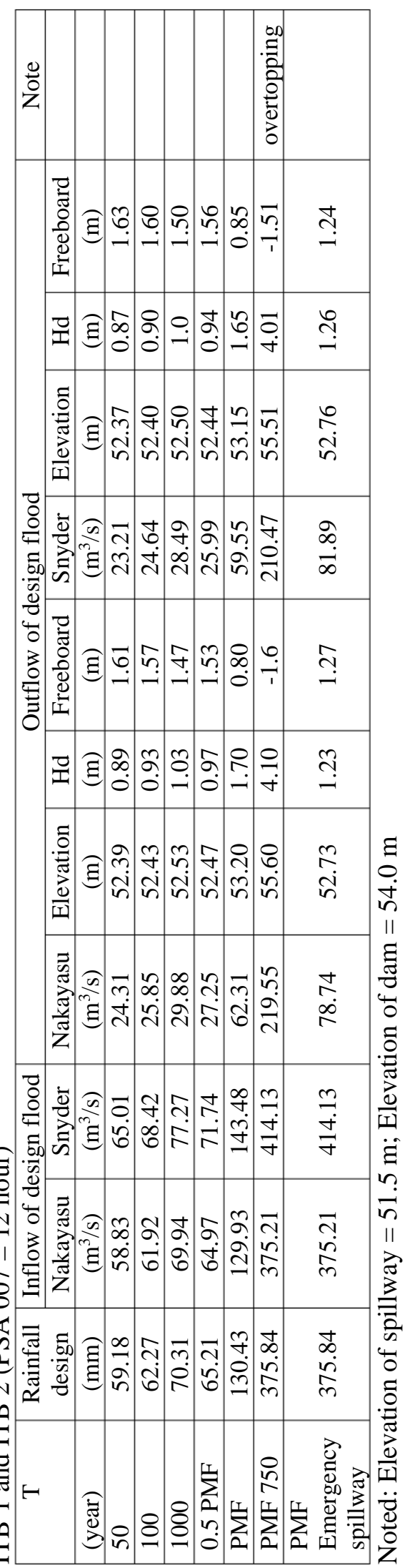


Based on the results of the flood discharge analysis above it can be seen that the condition of the Dam is safe against flood discharge of 0.5x QPMF and below. However, it is not safe against $\mathrm{Q}_{\mathrm{PMF}}$ discharge, overtopping will occur, and the watch height is less than the required minimum of $0.75 \mathrm{~m}$. However, if an emergency spillway is utilized, the condition is still safe from QPMF flooding. Based on field conditions, the emergency spillway is tending to solidify because it is used as a traffic access for residents in the fields/rice fields.

\section{2. Reservoir Capacity}

Based on the results of the latest bathymetry measurements, it is known that the reservoir volume at Normally Water Level (NWL) is 1,764,897 $\mathrm{m}^{3}$. While based on the results of a previous study in 1999 from PT.Indrakarya [2] the volume of reservoir was $2,293,800 \mathrm{~m}^{3}$, this shows that there was a reduction in sediment by 0.529 million $\mathrm{m}^{3}$, within a period of 19 years or sediment deposition of $27.84 \mathrm{~m}^{3} /$ year. The curved graph of the Banyukuwung Reservoir Capacity results of the latest bathymetry measurements are as follows Figure 4.

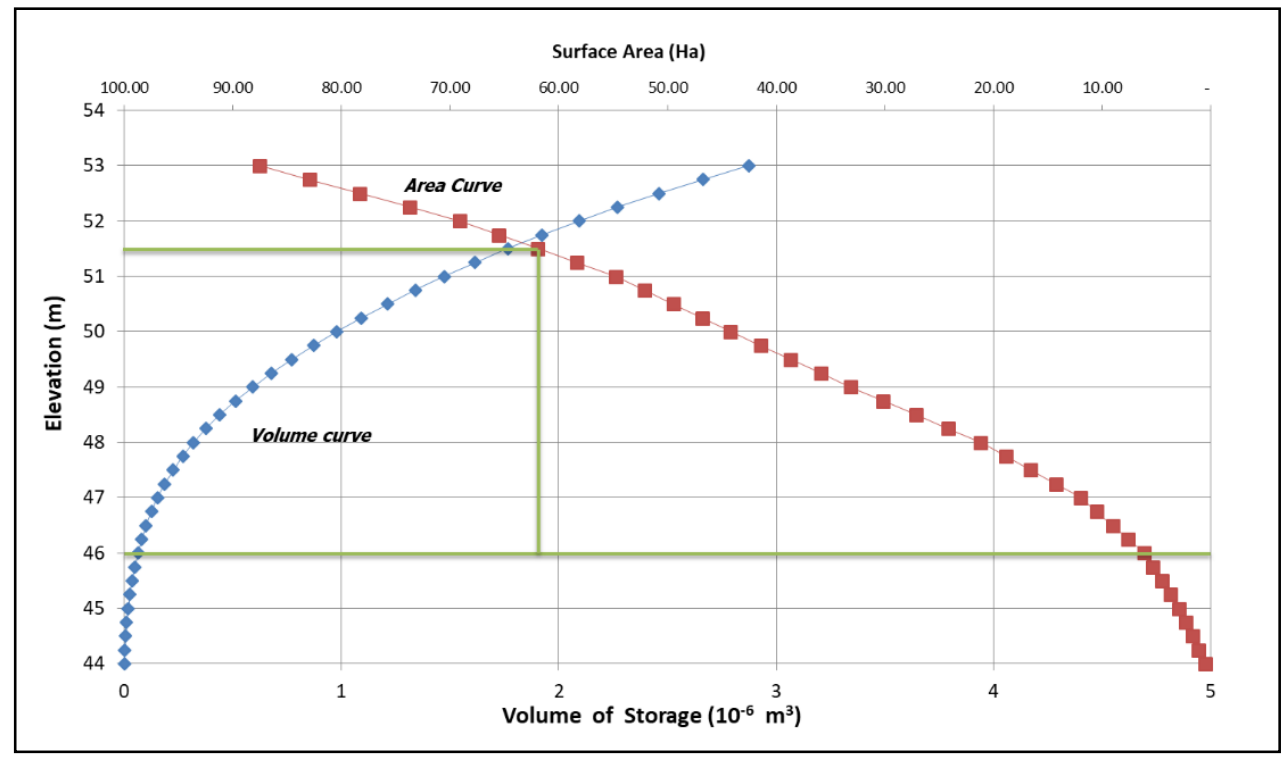

Figure 4. Reservoir capacity of Banyukuwung Dam on 2018

\section{3. Dam Stability Analysis}

\subsubsection{Geotechnical properties}

Banyukuwung Dam is a type of landfill with material in the form of grayish yellow clay to blackish gray with $\mathrm{N}$ values between $9 / 30 \mathrm{~cm}$ to $16 / 30 \mathrm{~cm}$. Whereas in bedrock $\mathrm{N}$ values range from $45 / 30 \mathrm{~cm}$ to above $60 / 30 \mathrm{~cm}$. Permeability test shows that the pile material is waterproof, with k values ranging from $10^{-5}$ to $10^{-8} \mathrm{~cm} / \mathrm{s}$. In bedrock k values range from $10^{-6}$ to $10^{-9} \mathrm{~cm} / \mathrm{sec}$. The results of laboratory analysis of soil mechanics show that the embankment has a specific gravity of 2.3 to 2.7 with a content weight of $1.51-2.07 \mathrm{gr} / \mathrm{cc}$. Atterberg limit shows LL value of $60.8 \%-69.9 \%$, PL value $=17.6 \%$ $25.8 \%$, and PI value $=40.4 \%-44.1 \%$, so it is included in the ML group (Silt- Low Plasticity). Consolidated Undrained (CU) triaxial test results the value of $C^{\prime}=0.14-0.19 \mathrm{~kg} / \mathrm{cm}^{2}$ and $\phi=30^{\circ}-31^{\circ}$. While the Unconsolidated Undrained (UU) triaxial results of C $=0.16-0.26 \mathrm{~kg} / \mathrm{cm}^{2}$ and $\phi=22^{\circ}-29^{\circ}$ while the consolidation parameters, $C_{c}=0.2498-0.3019$. The area around the pool has hilly morphology with lithology of clay, silt, silty clay and clayey silt which is mostly agricultural land. 


\subsubsection{Seepage Analysis of dams}

The amount of seepage that comes out of the Dam body is calculated by finite element in the form of Flux, namely the discharge (Q) of the seepage that passes through the dam core [7]. The seepage analysis analyzed was at the Flood Water Level (FWL El. $+52.5 \mathrm{~m}$ ), Normal Water Level (NWL El $+51.5 \mathrm{~m}$ ), and Lowest Water Level (LWL El. +42 m) shown if Figure $3-5$.

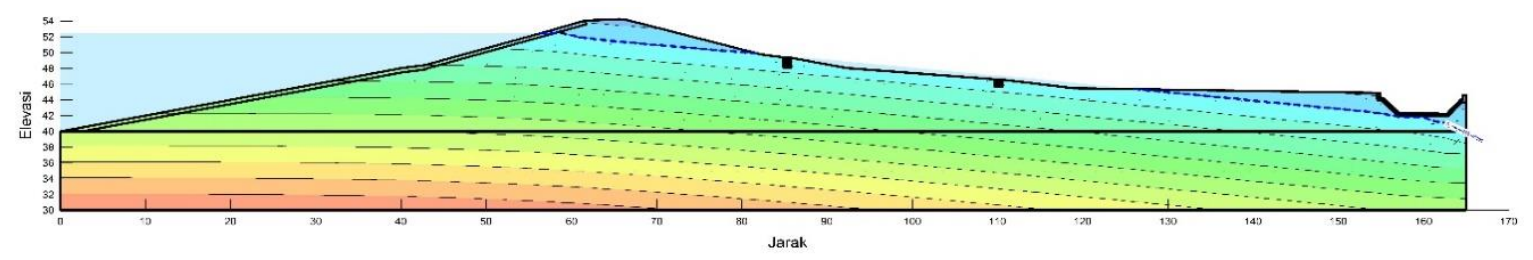

Figure 5. Seepage through Dam Body in FWL Conditions

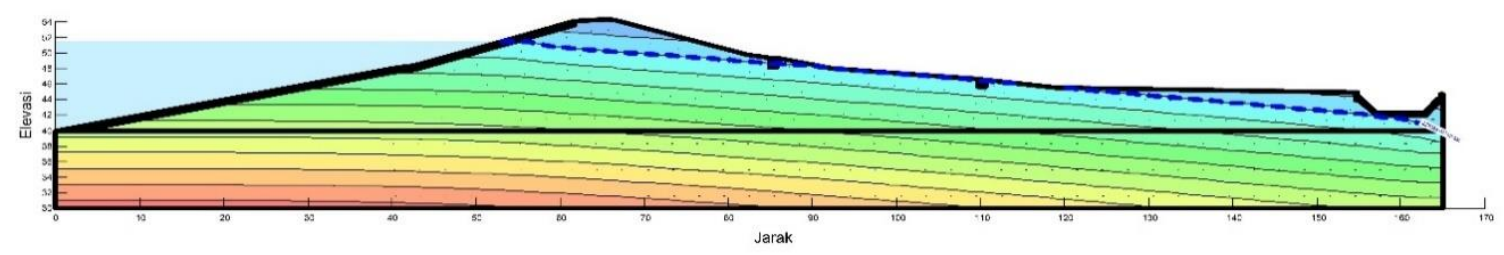

Figure 6. Dam Body Seepage in NWL Conditions

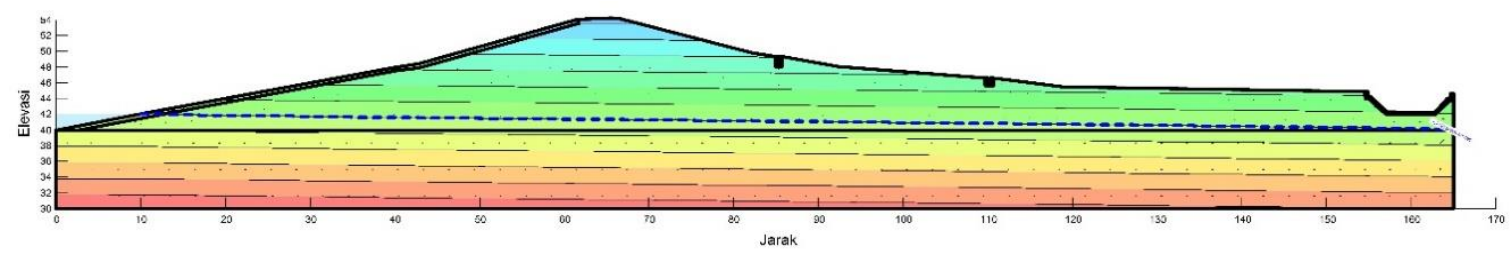

Figure 7. Dam Body Seepage in LWL Conditions

The results of seepage analysis on water level conditions are presented as follows:

$\mathrm{FWL}+52.5 \mathrm{~m}=9.36 \times 10^{-9} \mathrm{~m}^{3} / \mathrm{s} / \mathrm{m}$

$\mathrm{NWL}+51.5 \mathrm{~m}=8.56 \times 10^{-9} \mathrm{~m}^{3} / \mathrm{s} / \mathrm{m}$

$\mathrm{LWL}+42 \mathrm{~m}=1.11 \times 10^{-9} \mathrm{~m}^{3} / \mathrm{s} / \mathrm{m}$

Average $=6.34 \times 10^{-9} \mathrm{~m}^{3} / \mathrm{s} / \mathrm{m} \times 352 \mathrm{~m}=2.23 \times 10^{-6} \mathrm{~m}^{3} / \mathrm{s}$

Average seepage capacity $\left(2.23 \times 10^{-6} \mathrm{~m}^{3} / \mathrm{s}\right)<1 \%$ of the average $\mathrm{Q}$ of river inflow $\left(1.69 .10^{-4}\right.$ $\left.\mathrm{m}^{3} / \mathrm{s}\right)$. Therefore, it can be seen the seepage capacity that occurs in the foundation and body of the Banyukuwung Dam still meets the requirements specified:

- $\mathrm{Q}$ average river inflow $=0.016905 \mathrm{~m}^{3} / \mathrm{s}$

- $1 \%$ of the average river $\mathrm{Q}=1.69 .10^{-4} \mathrm{~m}^{3} / \mathrm{s}$.

\subsubsection{Slope Stability Analysis}

Dam slope stability was analyzed using the Bishop Modification method produced as shown in Figure 8, for Optimum Base Eartquake (OBE) conditions with 100 years earthquake return period. The calculation results are then tabulated as follows. 


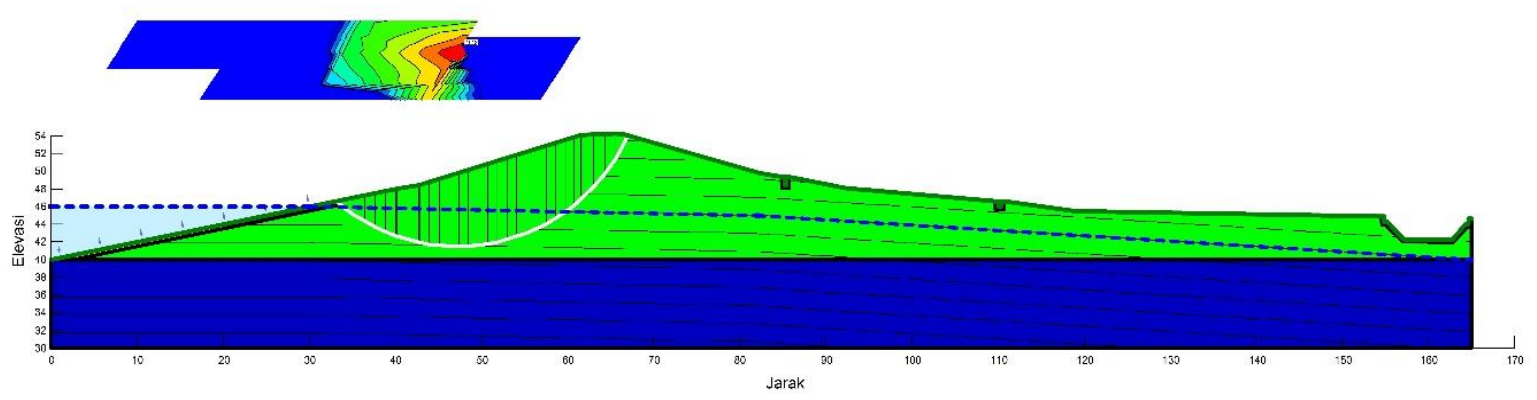

Figure 8. Analysis of the dam slope stability with OBE earthquake load (Return periode $\mathrm{T}=100$ years)

Table 5. Safety factor of OBE condition - water level (Upstream,Us/Downstream,Ds)

\begin{tabular}{|c|c|c|c|c|c|c|}
\hline \multirow[b]{2}{*}{ Condition of dam } & \multirow[b]{2}{*}{ FS } & \multicolumn{4}{|c|}{ Safety Factor } & \multirow[b]{2}{*}{ Note } \\
\hline & & $\begin{array}{c}\mathrm{y} / \mathrm{H}= \\
0.25\end{array}$ & $\begin{array}{c}\mathrm{y} / \mathrm{H}= \\
0.5\end{array}$ & $\begin{array}{c}\mathrm{y} / \mathrm{H}= \\
0.75\end{array}$ & $\mathrm{y} / \mathrm{H}=1$ & \\
\hline Us-empty & 1.1 & 2.46 & 2.53 & 2.57 & 2.60 & safe \\
\hline Ds-empty & 1.1 & 2.64 & 2.73 & 2.78 & 2.81 & safe \\
\hline Us -LWL & 1.1 & 2.16 & 2.22 & 2.25 & 2.28 & safe \\
\hline Ds-LWL & 1.1 & 2.60 & 2.68 & 2.72 & 2.75 & safe \\
\hline Us-NWL & 1.1 & 2.41 & 2.51 & 2.56 & 2.61 & safe \\
\hline Ds-NWL & 1.1 & 2.00 & 2.07 & 2.10 & 2.14 & safe \\
\hline Us-FWL & 1.1 & 2.62 & 2.75 & 2.81 & 2.88 & safe \\
\hline Ds-FWL & 1.1 & 1.85 & 1.91 & 1.95 & 1.97 & safe \\
\hline
\end{tabular}

Results of dam slope stability analysis with Maximum Design Earthquake (MDE) - T $=3000 \mathrm{yr}$ with Earthquake Map of the 2010, as follows Table 6.

Table 6. Safety factor of MDE condition - water level (Upstream,Us/Downstream,Ds)

\begin{tabular}{|c|c|c|c|c|c|c|}
\hline \multirow[b]{2}{*}{ Condition of Dam } & \multirow[b]{2}{*}{ FS } & \multicolumn{4}{|c|}{ Safety Factor } & \multirow[b]{2}{*}{ Note } \\
\hline & & $\begin{array}{c}\mathrm{y} / \mathrm{H}= \\
0.25\end{array}$ & $\mathrm{y} / \mathrm{H}=0.5$ & $\begin{array}{c}\mathrm{y} / \mathrm{H}= \\
0.75\end{array}$ & $\mathrm{y} / \mathrm{H}=1$ & \\
\hline Us-empty & 1.1 & 1.33 & 1.46 & 1.53 & 1.61 & safe \\
\hline Ds-empty & 1.1 & 1.31 & 1.46 & 1.54 & 1.62 & safe \\
\hline Us -LWL & 1.1 & 1.18 & 1.29 & 1.34 & 1.41 & safe \\
\hline Ds-LWL & 1.1 & 1.33 & 1.48 & 1.55 & 1.64 & safe \\
\hline Us-NWL & 1.1 & 1.10 & 1.28 & 1.28 & 1.36 & safe \\
\hline Ds-NWL & 1.1 & $0.98^{*}$ & 1.09 & 1.15 & 1.21 & Not Safe \\
\hline Us-FWL & 1.1 & 1.11 & 1.24 & 1.31 & 1.40 & safe \\
\hline Ds-FWL & 1.1 & $0.911^{*}$ & 1.01 & 1.07 & 1.13 & Not safe \\
\hline
\end{tabular}

Based on the table above it can be seen that the NWL and FWL conditions, with a ratio of $y / H=$ 0.25 , the downstream slope conditions are less safe (critical) in the earthquake return period $=3000$. Results of dam slope stability analysis with Maximum Design Earthquake (MDE) - T $=3000 \mathrm{yr}$ with Map of the 2017 Earthquake, as follows Table 7. 
Table 7. Safety factor of OBE condition - water level (Upstream,Us/Downstream,Ds)

\begin{tabular}{|c|c|c|c|c|c|c|}
\hline \multirow[b]{2}{*}{ Condition of Dam } & \multirow[b]{2}{*}{ FS } & \multicolumn{4}{|c|}{ Safety Factor } & \multirow[b]{2}{*}{ Noted } \\
\hline & & $\begin{array}{c}\mathrm{y} / \mathrm{H}= \\
0.25\end{array}$ & $\mathrm{y} / \mathrm{H}=0.5$ & $\begin{array}{c}\mathrm{y} / \mathrm{H}= \\
0.75\end{array}$ & $\mathrm{y} / \mathrm{H}=1$ & \\
\hline Us-empty & 1.1 & 1.46 & 1.60 & 1.66 & 1.74 & safe \\
\hline Ds-empty & 1.1 & 1.46 & 1.61 & 1.69 & 1.77 & safe \\
\hline Us -LWL & 1.1 & 1.29 & 1.40 & 1.46 & 1.52 & safe \\
\hline Ds-LWL & 1.1 & 1.48 & 1.63 & 1.70 & 1.78 & safe \\
\hline Us-NWL & 1.1 & 1.29 & 1.35 & 1.42 & 1.50 & safe \\
\hline Ds-NWL & 1.1 & 1.10 & 1.21 & 1.27 & 1.33 & safe \\
\hline Us-FWL & 1.1 & 1.24 & 1.39 & 1.46 & 1.55 & safe \\
\hline Ds-FWL & 1.1 & $1.02^{*}$ & 1.12 & 1.17 & 1.23 & Not safe \\
\hline
\end{tabular}

Based on the table above, it can be seen that in the FWL condition, with a ratio of $y / H=0.25$, the downstream slope condition is less safe (critical) in the earthquake return period $=3000 \mathrm{yr}$

\section{Conclusions}

Based on the results of the analysis above on the stability conditions of the Banyukuwung Dam, it can be concluded as follows:

Under normal load conditions, the hydrological and flood hydraulic conditions are in the "sufficient" category, the seepage condition is safe and the review of the body structure aspects of the dam is "safe". Whereas in terms of dynamic (extraordinary) load conditions, the hydrological and flood hydraulic conditions of the category of "less", seepage conditions are safe, while the condition of the dam's body structure is "less safe". Consequently, the safety status of the dam is "ENOUGH", but there is a threat to the hydrological/hydraulics conditions at the QPMF discharge potentially overtopping. While the stability of the dam slope there is a threat to the Maximum Design Earthquake (MDE), $T=3000$ year dynamic load conditions. However, if we look at the design criteria for the Banyukuwung Dam, it was originally designed according to the small pond criteria, so that the above conditions are quite understandable that if a safety review is carried out based on the criteria of a large dam, there are a number of parameters that need to be adjusted accordingly.

\section{Acknowledgements}

Thank you to the Faculty of Engineering Universitas Brawijaya, which has provided research opportunities and also to the head of the Pemali Juana River Region Center, especially the operation and maintenance work unit that has supported the writing of this research.

\section{References}

[1] IPCC., 2007 Intergovernmental Panel on Climate Change, IPCC Fourth Assessment Report: Climate Change

[2] Ammar Rouaiguia and Mohammed A. Dahim, 2013. Numerical Modeling of Slope Stability Analysis, International Journal of Engineering Science and Innovative Technology (IJESIT), ISSN: 2319-5967

[3] Torimtubun, Angelina Tutulenan, 2018. Analisa Banjir Akibat Keruntuhan Bendungan Banyukuwung dengan Menggunakan HEC-RAS. Undergraduate thesis, Universitas Brawijaya.

[4] Anonymous, 2004. Stability analysis of earth fill dams due to earthquake load. Guidelines of PdT14-2004. Ministry Public Work and Transport.

[5] Anonymous, 2003. Guidelines Inspection/Evaluation Dam and the Guidelines for Common Criteria Dam Design, SK Dirjen SDA/KKB No.05 / KPTS / 2003. Dam Safety Commission. 
[6] Asmaranto, R., Suryono, A., \& Hidayat, M. 2019. Inspections of Hydro-Geotechnical on Ngancar Dam. Civil and Environmental Science Journal, 2(2), pp.117-127. doi:https://doi.org/10.21776/ub.civense.2019.00202.5

[7] Asmaranto, runi et.al. 2020. Safety evaluation of the existing Grawan Dam based on hydrogeotechnical behaviour conditions to ensure the availability of water resources, published under licence by IOP Publishing Ltd, IOP Conference Series: Earth and Environmental Science, Volume 437, The 3rd International Conference of Water Resources Development and Environmental Protection 12-13 October 2019, Malang https://iopscience.iop.org/article/10.1088/1755-1315/437/1/012006,

[8] E Fadaei Kermani and G. A Barani. 2012. Seepage Analysis through Earth Dam Based on Finite Difference Method, Journal of Basic and Applied Scientific Research ISSN 2090-4304 vol 2 (11) page 11621-11625

[9] Fahim Ashkar and Taha B. M. J. Ouarda, Approximate Confidence Intervals for Quantiles of Gamma and Generalized Gamma Distributions, Journal of Hydrologic Engineering, 10.1061/(ASCE)1084-0699(1998)3:1(43), 3, 1, (43-51), (1998).

[10] J.R. Swaisgood P.E. 2003. Embankment dam deformations caused by earthquakes. Colorado. USA

[11] Ms Abhilasha P.S and T.G Antony Balan. 2013. Numerical Analysis of Seepage in Embankment Dams, IOSR Journal of Mechanical and Civil Engineering (IOSR-JMCE) e-ISSN: 2278-1684, p-ISSN: 2320-334X PP 13-23

[12] PR. Bamane and Dr.S.S.Valunjkar. 2014. Dam Safety Instrumentation, American Journal of Engineering Research (AJER) e-ISSN: 2320-0847 p-ISSN: 2320-0936 page 58-62

[13] Sosrodarsono, Suyono \& Takeda, Kensaku. 1989. Bendungan Type Urugan. Jakarta: Erlangga.

[14] Xiaoli Ding and Hui Qin. 1998. Geotechnical Instruments in Structural Monitoring, Journal of Geospatial Engineering, Vol. 2, No.2, pp.45-56

[15] Yu Zhao, Zi-Yhi Tong and Qing Lu. 2014. Slope Stability Analysis Using Slice-Wise Factor of Safety, Hindawi Publishing Corporation, Mathematical Problems in Engineering, Volume 2014, Article ID 712145, 6 pages.

[16] Virgiawan Eric A, etal. 2014. Analisa Keruntuhan Bendungan Gondang Dengan Menggunakan Program Zhong Xing HY21. Jurnal Teknik Pengairan

[17] Harto, Sri BR. 2009. Analisa Hidrologi. Yogyakarta: Biro Penerbit Keluarga Mahasiswa UGM

[18] Soemarto, C.D. Hidrologi teknik. 1995. Jakarta: Erlangga 